DEMONSTRATIO MATHEMATICA

Vol. XLVII No $2 \quad 2014$

\author{
Yu. M. Movsisyan, V. A. Aslanyan
}

\title{
DE MORGAN FUNCTIONS AND FREE DE MORGAN ALGEBRAS
}

\begin{abstract}
It is commonly known that the free Boolean algebra on $n$ free generators is isomorphic to the Boolean algebra of Boolean functions of $n$ variables. The free bounded distributive lattice on $n$ free generators is isomorphic to the bounded lattice of monotone Boolean functions of $n$ variables. In this paper, we introduce the concept of De Morgan function and prove that the free De Morgan algebra on $n$ free generators is isomorphic to the De Morgan algebra of De Morgan functions of $n$ variables. This is a solution of the problem suggested by B. I. Plotkin.
\end{abstract}

\section{Introduction and preliminaries}

An algebra $(Q ;\{+, \cdot,-, 0,1\})$ with two binary, one unary and two nullary operations is called a De Morgan algebra if $(Q ;\{+, \cdot, 0,1\})$ is a bounded distributive lattice with least element 0 and greatest element 1 and $\left(Q ;\left\{+, \cdot,^{-}, 0,1\right\}\right)$ satisfies the following identities:

$$
\begin{gathered}
\overline{x+y}=\bar{x} \cdot \bar{y}, \\
\overline{\bar{x}}=x,
\end{gathered}
$$

where $\overline{\bar{x}}=\overline{(\bar{x})}([2,4,6,8,18,19,24,31,32,34 ;$; In book $[6]$, the definition of the reduct of De Morgan algebra is given). The standard fuzzy algebra $F=([0,1] ; \max (x, y), \min (x, y), 1-x, 0,1)$ is an example of a De Morgan algebra.

Except in mathematical logic $([1,3,7,14,15,20,22,23])$ and algebra, De Morgan algebras (and De Morgan bisemilattices) have applications in multi-valued simulations of digital circuits too $(9,10])$.

A De Morgan algebra $\mathcal{F}=\left(F ;\left\{+, \cdot,{ }^{-}, 0,1\right\}\right)$ is called a free De Morgan algebra with the system of free generators $X \subseteq F$ if the algebra $\mathcal{F}$ is generated by the subset $X \subseteq F$ and for every De Morgan algebra $\mathfrak{S}=\left(S ;\left\{+, \cdot{ }^{-}, 0,1\right\}\right)$

2010 Mathematics Subject Classification: 06B25, 06D30, 06E30, 06B20, 08B20.

Key words and phrases: Antichain, De Morgan function, free De Morgan algebra, monotone Boolean function, disjunctive (conjunctive) normal form of De Morgan function. 
and for every mapping $\mu: X \rightarrow S$, there exists a unique homomorphism: $\nu: \mathcal{F} \rightarrow \mathfrak{S}$ with $\left.\nu\right|_{X}=\mu$. The concepts of the free bounded distributive lattice and the free Boolean algebra have similar definitions $([16])$. These definitions are special cases of the general concept of the free algebra of variety ([17, 35]).

Let us consider the following De Morgan algebras: $\mathbf{2}=(\{0,1\}$; $\left.\left\{+, \cdot,^{-}, 0,1\right\}\right), \mathbf{3}=\left(\{0, a, 1\} ;\left\{+, \cdot,^{-}, 0,1\right\}\right)$, where $\bar{a}=a$, and $\mathbf{4}=$ $\left(\{0, a, b, 1\} ;\left\{+, \cdot,^{-}, 0,1\right\}\right)$, where $\bar{a}=a, \bar{b}=b, a+b=1, a \cdot b=0$. (Here 0 and 1 are respectively the least and greatest elements of distributive lattice and so the other values of operations are defined uniquely. In particular, $\overline{1}=0, \overline{0}=1$, for those three algebras.)

Let us recall the following result that makes clear our approach for the definition of the concept of De Morgan functions.

THEOREM 1.1. ([18]) Every non-trivial subdirectly irreducible De Morgan algebra is isomorphic to one of the following algebras: $\mathbf{2 , 3 , 4}$, where $\mathbf{2}$ is the unique non-trivial subdirectly irreducible Boolean algebra.

As a corollary, we can state that the free $n$-generated De Morgan algebra is isomorphic to the subalgebra of $\mathbf{4}^{\mathbf{4}^{n}}$ generated by the projections. Elements of this subalgebra are functions from $4^{n}$ into 4 .

Let $B=\{0,1\}$. Define the operations,$+ \cdot{ }^{-}$on $B$ by the following way: $0+0=0,0+1=1+0=1+1=1,0 \cdot 1=0 \cdot 0=1 \cdot 0=0,1 \cdot 1=$ $1, \overline{0}=1, \overline{1}=0$. We get the Boolean algebra $\mathbf{2}=\left(B ;\left\{+, \cdot{ }^{-}, 0,1\right\}\right)$ and the bounded distributive lattice $(B ;\{+, \cdot, 0,1\})$. Let " $\leq$ " be its order. For $u=\left(u_{1}, \ldots, u_{n}\right), v=\left(v_{1}, \ldots, v_{n}\right) \in B^{n}$, we define: $u \leq v$ if and only if $u_{i} \leq v_{i}$ for all $i=1, \ldots, n$, where $B^{n}$ is the set of all $n$-element sequences of $B$. Here and afterwards $n \geq 1$ is a positive integer.

Definition 1.2. A Boolean function $f: B^{n} \rightarrow B$ is called monotone if

$$
x \leq y \Rightarrow f(x) \leq f(y),
$$

where $x, y \in B^{n}$.

If $u=\left(u_{1}, \ldots, u_{n}\right), v=\left(v_{1}, \ldots, v_{n}\right) \in B^{n}$ then we will say $u \preceq v$ if there exists $k(1 \leq k \leq n)$ such that $u_{i}=v_{i}$ for all $i \neq k$ and $u_{k}=0, v_{k}=1$. It is easy to see that a Boolean function $f: B^{n} \rightarrow B$ is monotone if and only if

$$
x \preceq y \Rightarrow f(x) \leq f(y), x, y \in B^{n} .
$$

Denote the set of all monotone Boolean functions of $n$ variables by $\mathcal{M}_{n}$. We can define $f+g$ and $f \cdot g$ for any two Boolean functions of $n$ variables by the standard way: $(f+g)(x)=f(x)+g(x),(f \cdot g)(x)=f(x) \cdot g(x), x \in B^{n}$. It is obvious that if $f$ and $g$ are monotone Boolean functions, then $f+g$ and $f \cdot g$ 
are monotone, too. Thus, we get the algebra $\mathfrak{L}_{n}=\left(\mathcal{M}_{n} ;\{+, \cdot, 0,1\}\right)$ (here 0 and 1 are the constant Boolean functions) which obviously is a bounded distributive lattice. Also, let $m_{n}=\left|\mathcal{M}_{n}\right|$ be the number of monotone Boolean functions of $n$ variables. (Note that the numbers $m_{n}$ are called Dedekind's numbers.) For instance, $m_{1}=3, m_{2}=6, m_{3}=20, m_{4}=168, m_{5}=$ $7581, m_{6}=7828354([11,[21])$.

Now, let $S \subseteq 2^{\{1, \ldots, n\}}$ be an antichain (or Sperner set 12,36 ) with respect to the order $\subseteq$. This means that $S$ consists of subsets of $\{1, \ldots, n\}$, none of which is contained in any other subset from $S$. Note that the empty set is also considered an antichain. For an antichain $S \subseteq 2^{\{1, \ldots, n\}}$ define the following monotone Boolean function:

$$
f_{S}\left(x_{1}, \ldots, x_{n}\right)=\sum_{s \in S} \prod_{i \in s} x_{i} .
$$

For $S=\emptyset$ we set $f_{\emptyset}=0$, and for $S=\{\emptyset\}$ we set $f_{\{\emptyset\}}=1$. Notice that $f_{S}$ does not depend on the order of the elements in the set $S$. It is easy to see that if $S_{1} \neq S_{2}$ are two antichains then $f_{S_{1}} \neq f_{S_{2}}$. To see this without loss of generality suppose that there exists $s \in S_{1}$ such that $s \notin S_{2}$. We can also suppose that there does not exist $s^{\prime} \in S_{2}$ with $s^{\prime} \subseteq s$. Otherwise, we would take $s^{\prime}$ instead of $s$ (in that case $s^{\prime} \notin S_{1}$, because $S_{1}$ is an antichain). Take the following values of the variables:

$$
x_{i}= \begin{cases}1, & \text { if } i \in s, \\ 0, & \text { if } i \notin s .\end{cases}
$$

For these values of variables, we have: $f_{S_{1}}=1$ and $f_{S_{2}}=0$.

The form 1.1 is uniquely determined by the antichain $S \subseteq 2^{\{1, \ldots, n\}}$. And conversely, we show next that every monotone Boolean function is obtained in that way.

We prove the following well-known result since we use that proof in Section 3 .

Proposition 1.3. (|6, 11, 12, 16, 21, 33, 36|) For every monotone Boolean function of $n$ variables, there exists a unique antichain $S \subseteq 2^{\{1, \ldots, n\}}$ such that $f=f_{S}$.

Proof. For $a=\left(a_{1}, \ldots, a_{n}\right) \in B^{n}$ let $s_{a}=\left\{i: a_{i}=1\right\}$. Consider the set $A=\left\{s_{a}: a \in B^{n}, f(a)=1\right\}$. Let $S$ be the subset of $A$, consisting exactly of all minimal sets in $A$. Then $S \subseteq 2^{\{1, \ldots, n\}}$ is an antichain. Notice that $f\left(a_{1}, \ldots, a_{n}\right)=1$ iff for some $s \in S$, we have $a_{i}=1$ for all $i \in s$. The same is valid for $f_{S}$. Therefore, $f\left(a_{1}, \ldots, a_{n}\right)=f_{S}\left(a_{1}, \ldots, a_{n}\right)$ for all $\left(a_{1}, \ldots, a_{n}\right) \in B^{n}$, and so $f=f_{S}$. The uniqueness follows from the argument stated above. 
Define the Boolean functions:

$$
\delta_{n}^{i}\left(x_{1}, \ldots, x_{n}\right)=x_{i}, i=1, \ldots, n .
$$

Theorem 1.4. ([6, 12, 16, 33, 36]) The algebra $\mathfrak{L}_{n}$ is the free bounded distributive lattice with the system of free generators: $\Delta=\left\{\delta_{n}^{1}, \ldots, \delta_{n}^{n}\right\}$. Hence every free n-generated bounded distributive lattice is isomorphic to the bounded distributive lattice $\mathfrak{L}_{n}$.

The problem of similar characterization of the $n$-generated free De Morgan algebra is suggested by B. I. Plotkin (in algebraic conference, St Petersburg, Russia, 1981). In this paper, we introduce the concept of De Morgan function and prove that the free De Morgan algebra on $n$ free generators is isomorphic to the De Morgan algebra of De Morgan functions of $n$ variables.

Now, let us establish some further properties of monotone Boolean functions, which are used in the third section.

If $i \in\{1, \ldots, 2 n\}$, then denote:

$$
\sigma(i)= \begin{cases}i+n, & \text { if } 1 \leq i \leq n, \\ i-n, & \text { if } n+1 \leq i \leq 2 n\end{cases}
$$

For a monotone Boolean function $f\left(x_{1}, \ldots, x_{2 n}\right)=\sum_{s \in S} \prod_{i \in s} x_{i}$ consider the function:

$$
f^{\prime}\left(x_{1}, \ldots, x_{2 n}\right)=\prod_{s \in S} \sum_{i \in s} x_{\sigma(i)} .
$$

Clearly $f^{\prime}$ is also a monotone Boolean function.

LEMMA 1.5. For any monotone Boolean function $f$ of $2 n$ variables, the following equality holds for all $u_{1}, \ldots, u_{n}, v_{1}, \ldots, v_{n} \in B$ :

$$
\overline{f\left(u_{1}, \ldots, u_{n}, \bar{v}_{1}, \ldots, \bar{v}_{n}\right)}=f^{\prime}\left(v_{1}, \ldots, v_{n}, \bar{u}_{1}, \ldots, \bar{u}_{n}\right) .
$$

Proof. For $i=1, \ldots, 2 n$ define:

$$
t_{i}= \begin{cases}u_{i}, & \text { if } 1 \leq i \leq n, \\ \bar{v}_{i-n}, & \text { if } n+1 \leq i \leq 2 n .\end{cases}
$$

Then we have:

$$
\begin{aligned}
& \overline{f\left(u_{1}, \ldots, u_{n}, \bar{v}_{1}, \ldots, \bar{v}_{n}\right)}=\overline{\sum_{s \in S} \prod_{i \in s} t_{i}}=\prod_{s \in S} \sum_{i \in s} \bar{t}_{i}= \\
& f^{\prime}\left(\bar{t}_{\sigma(1)}, \ldots, \bar{t}_{\sigma(n)}, \bar{t}_{\sigma(n+1)}, \ldots, \bar{t}_{\sigma(2 n)}\right)=f^{\prime}\left(v_{1}, \ldots, v_{n}, \bar{u}_{1}, \ldots, \bar{u}_{n}\right) .
\end{aligned}
$$

The lemma is proved.

\section{De Morgan functions}

Denote $D=B \times B=\{(0,0),(1,0),(0,1),(1,1)\}=\{0, a, b, 1\}$, where $0=(0,0), a=(1,0), b=(0,1), 1=(1,1)$. Defining $0+x=x+0=x$ and 
$1 \cdot x=x \cdot 1=x$ for all $x \in D$, and $a+b=b+a=1, a \cdot b=b \cdot a=0, \overline{0}=1$, $\overline{1}=0, \bar{a}=a, \bar{b}=b$, we get the De Morgan algebra $4=\left(D ;\left\{+, \cdot,{ }^{-}, 0,1\right\}\right)$. Notice that

$$
\begin{aligned}
\overline{(u, v)} & =(\bar{v}, \bar{u}), \\
\left(u_{1}, v_{1}\right)+\left(u_{2}, v_{2}\right) & =\left(u_{1}+u_{2}, v_{1}+v_{2}\right), \\
\left(u_{1}, v_{1}\right) \cdot\left(u_{2}, v_{2}\right) & =\left(u_{1} \cdot u_{2}, v_{1} \cdot v_{2}\right),
\end{aligned}
$$

(here the operations on the right hand side are the operations of the Boolean algebra 2). For $x \in D$ let

$$
x^{*}= \begin{cases}x, & \text { if } x=0,1, \\ a, & \text { if } x=b, \\ b, & \text { if } x=a\end{cases}
$$

Also for $c=\left(c_{1}, \ldots, c_{n}\right), d=\left(d_{1}, \ldots, d_{n}\right) \in D^{n}$ we say that $d$ is a permitted modification of $c$ if for some $k(1 \leq k \leq n)$, we have $d_{i}=c_{i}$ for all $1 \leq i \leq n, i \neq k$ and

$$
d_{k}= \begin{cases}a, & \text { if } c_{k}=0, \\ 1, & \text { if } c_{k}=b\end{cases}
$$

Definition 2.1. A function $f: D^{n} \rightarrow D$ is called a De Morgan function if the following conditions hold:

(1) if $x_{i} \in\{0,1\}, i=1, \ldots, n$ then $f\left(x_{1}, \ldots, x_{n}\right) \in\{0,1\}$,

(2) if $x_{i} \in D, i=1, \ldots, n$ then $f\left(x_{1}^{*}, \ldots, x_{n}^{*}\right)=\left(f\left(x_{1}, \ldots, x_{n}\right)\right)^{*}$,

(3) if $x, y \in D^{n}$ with $f(x) \neq b$ and $y$ is a permitted modification of $x$ then $f(y) \in\{f(x), a\}$.

Notice that Condition (1) is a consequence of Condition (2), however it is convenient to write it as a separate condition.

Note that it follows from Condition (1) that every De Morgan function is an extension of some Boolean function. And notice that the constant functions $f=1$ and $f=0$ are De Morgan functions, but the constant functions $f=a$ and $f=b$ are not. This means that 0 and 1 are the only constant De Morgan functions. Further examples of De Morgan functions are $f(x)=x, g(x)=\bar{x}, h(x, y)=x \cdot y, q(x, y)=x+y$, where the operations on the right hand side are the operations of the De Morgan algebra 4 . We can straightforwardly verify that those functions satisfy the Conditions (1) - (3) of Definition 2.1, but it also follows from the results of the next section.

As Boolean functions, De Morgan functions (and also all functions $\left.D^{n} \rightarrow D\right)$ can be given by tables. Also note that there is an algorithm which, for a given table of a function $f: D^{n} \rightarrow D$, determines whether $f$ is 
a De Morgan function. Let us find the complexity of this algorithm depending on the number of the rows of the table. We denote that number by $k$ (obviously, $k=4^{n}$ ). To test whether a function $f$ is a De Morgan function we should check whether Condition (2) and (3) are satisfied for $f$ (as we mentioned above, Condition (1) is a consequence of Condition (2)). And it is easy to see that to check Condition (2) we need no more than $O(k)$ operations, and to verify Condition (3) we do no more than $O\left(k^{2}\right)$ operations, as we should consider the pairs of rows to decide whether one of them is a permitted modification of the other (we define $O(k)$ to be a function $p(k)$ such that the ratio $\frac{p(k)}{k}$ is bounded). And thus the complexity of algorithm is polynomial (more precisely, it is not greater than $C \cdot k^{2}$ for some constant $C$ ).

For $x_{i} \in D$, we denote by $\left(u_{i}, v_{i}\right)$ the pair from $B \times B$ which is equal to $x_{i}$, i.e. $x_{i}=\left(u_{i}, v_{i}\right)$. (Often we will consider $B=\{0,1\}$ as a subset of $D$.)

Definition 2.2. The function $f: D^{n} \rightarrow D$ is called a quasi-De Morgan function, if there exists a Boolean function $\varphi: B^{2 n} \rightarrow B$ such that

$$
f\left(x_{1}, \ldots, x_{n}\right)=\left(\varphi\left(u_{1}, \ldots, u_{n}, \bar{v}_{1}, \ldots, \bar{v}_{n}\right), \varphi\left(v_{1}, \ldots, v_{n}, \bar{u}_{1}, \ldots, \bar{u}_{n}\right)\right),
$$

for all $x_{1}, \ldots, x_{n} \in D$.

Proposition 2.3. The function $f: D^{n} \rightarrow D$ is a quasi-De Morgan function if and only if it satisfies Conditions (1) and (2) of Definition 2.1.

Proof. Let $f$ be a quasi-De Morgan function. If $x_{i} \in\{0,1\}$, then $u_{i}=v_{i}$ and $\varphi\left(u_{1}, \ldots, u_{n}, \bar{v}_{1}, \ldots, \bar{v}_{n}\right)=\varphi\left(v_{1}, \ldots, v_{n}, \bar{u}_{1}, \ldots, \bar{u}_{n}\right)$. Hence, $f\left(x_{1}, \ldots, x_{n}\right) \in$ $B$. Thus, Condition (1) holds for $f$. Now let us check Condition (2). To do this recall that $(u, v)^{*}=(v, u)$. Hence,

$$
\begin{aligned}
& f\left(x_{1}^{*}, \ldots, x_{n}^{*}\right)=\left(\varphi\left(v_{1}, \ldots, v_{n}, \bar{u}_{1}, \ldots, \bar{u}_{n}\right), \varphi\left(u_{1}, \ldots, u_{n}, \bar{v}_{1}, \ldots, \bar{v}_{n}\right)\right) \\
& \quad=\left(\varphi\left(u_{1}, \ldots, u_{n}, \bar{v}_{1}, \ldots, \bar{v}_{n}\right), \varphi\left(v_{1}, \ldots, v_{n}, \bar{u}_{1}, \ldots, \bar{u}_{n}\right)\right)^{*}=\left(f\left(x_{1}, \ldots, x_{n}\right)\right)^{*} .
\end{aligned}
$$

Now suppose Conditions (1) and (2) hold for $f$, and let us prove that there exists a Boolean function $\varphi$ with condition (2.1). First we prove that there are at most $2^{4^{n}}$ functions for which Conditions (1) and (2) hold. To see this notice that there are $2^{n} n$-tuples $\left(u_{1}, \ldots, u_{n}\right) \in B^{n}$. For such $n$ tuples $f$ can take only two values (by Condition (1)). Further, if the $n$ tuple $\left(v_{1}, \ldots, v_{n}\right) \in D^{n}$ contains $a$ or $b$, then $\left(v_{1}^{*}, \ldots, v_{n}^{*}\right) \neq\left(v_{1}, \ldots, v_{n}\right)$ and $f\left(v_{1}^{*}, \ldots, v_{n}^{*}\right)$ is uniquely determined by $f\left(v_{1}, \ldots, v_{n}\right)$ (by Condition $\left.(2)\right)$. There are $4^{n}-2^{n}$ such $n$-tuples. Thus, the number of such functions does not exceed $2^{2^{n}} \cdot 4^{\frac{4^{n}-2^{n}}{2}}=2^{2^{n}} \cdot 2^{4^{n}-2^{n}}=2^{4^{n}}$. It is clear that for a quasi-De Morgan function $f$, there exists exactly one Boolean function $\varphi$ with condition 2.1. Therefore, there are $2^{4^{n}}=2^{2^{2 n}}$ quasi-De Morgan functions. And all quasiDe Morgan functions satisfy Conditions (1) and (2). Hence, all functions $f: D^{n} \rightarrow D$ satisfying (1) and (2) are quasi-De Morgan functions. 
This proposition makes clear why those functions are called quasi-De Morgan functions. As we mentioned in the proof, for a quasi-De Morgan function $f: D^{n} \rightarrow D$, there exists a unique Boolean function $\varphi: B^{2 n} \rightarrow B$ which satisfies (2.1). To emphasize that $\varphi$ is the unique Boolean function corresponding to $f$, we denote it by $\varphi_{f}$.

THEOREM 2.4. The function $f: D^{n} \rightarrow D$ is a De Morgan function if and only if it is a quasi-De Morgan function and $\varphi_{f}$ is a monotone Boolean function.

Proof. If $f$ is a De Morgan function then by Proposition 2.3, it is a quasi-De Morgan function. Let us prove that $\varphi_{f}$ is monotone. Let $u=\left(u_{1}, \ldots, u_{2 n}\right)$, $v=\left(v_{1}, \ldots, v_{2 n}\right) \in B^{2 n}$ and for some $k(1 \leq k \leq 2 n) u_{i}=v_{i}$, if $i \neq k$, $u_{k}=0, v_{k}=1$. We show that $\varphi_{f}(u) \leq \varphi_{f}(v)$. Suppose it is not true, i.e. $\varphi_{f}(u)=1, \varphi_{f}(v)=0$. For $1 \leq i \leq n$ denote:

$$
c_{i}= \begin{cases}\left(u_{i}, \overline{u_{n+i}}\right), & \text { if } 1 \leq k \leq n, \\ \left(\overline{u_{n+i}}, u_{i}\right), & \text { if } n+1 \leq k \leq 2 n,\end{cases}
$$

and

$$
d_{i}= \begin{cases}\left(v_{i}, \overline{v_{n+i}}\right), & \text { if } 1 \leq k \leq n, \\ \left(\overline{v_{n+i}}, v_{i}\right), & \text { if } n+1 \leq k \leq 2 n .\end{cases}
$$

Suppose $1 \leq k \leq n$. Then $d=\left(d_{1}, \ldots, d_{n}\right)$ is a permitted modification of $c=\left(c_{1}, \ldots, c_{n}\right)$.

$$
\begin{aligned}
f(c) & =\left(\varphi_{f}\left(u_{1}, \ldots, u_{n}, u_{n+1}, \ldots, u_{2 n}\right), \varphi_{f}\left(\overline{u_{n+1}}, \ldots, \overline{u_{2 n}}, \bar{u}_{1}, \ldots, \bar{u}_{n}\right)\right) \\
& =\left(1, \varphi_{f}\left(\overline{u_{n+1}}, \ldots, \overline{u_{2 n}}, \bar{u}_{1}, \ldots, \bar{u}_{n}\right)\right) \neq b .
\end{aligned}
$$

Analogously:

$$
f(d)=\left(0, \varphi_{f}\left(\overline{v_{n+1}}, \ldots, \overline{v_{2 n}}, \bar{v}_{1}, \ldots, \bar{v}_{n}\right)\right) .
$$

By Condition (3), we have $f(d)=f(c)$ or $f(d)=a$. This gives a contradiction with the above equalities.

Now suppose $n+1 \leq k \leq 2 n$. Then $c$ is a permitted modification of $d$. We have:

$$
\begin{aligned}
f(d) & =\left(\varphi_{f}\left(\overline{v_{n+1}}, \ldots, \overline{v_{2 n}}, \bar{v}_{1}, \ldots, \bar{v}_{n}\right), \varphi_{f}\left(v_{1}, \ldots, v_{n}, v_{n+1}, \ldots, v_{2 n}\right)\right) \\
& =\left(\varphi_{f}\left(\overline{v_{n+1}}, \ldots, \bar{v}_{2 n}, \bar{v}_{1}, \ldots, \bar{v}_{n}\right), 0\right) \neq b .
\end{aligned}
$$

And also

$$
f(c)=\left(\varphi_{f}\left(\overline{u_{n+1}}, \ldots, \overline{u_{2 n}}, \bar{u}_{1}, \ldots, \bar{u}_{n}\right), 1\right) .
$$

Again, by Condition (3), we have $f(c)=f(d)$ or $f(c)=a$, which is a contradiction.

In both cases, we arrived at a contradiction. Consequently, $\varphi_{f}$ is a monotone Boolean function. 
Thus, the "only if"-part of the theorem is proved. Now, let us prove the "if"-part.

Suppose that $f$ is a quasi-De Morgan function and $\varphi_{f}$ is a monotone Boolean function. We verify that Condition (3) holds for $f$. To see this, let $d=\left(d_{1}, \ldots, d_{n}\right) \in D^{n}$ be a permitted modification of $c=\left(c_{1}, \ldots, c_{n}\right) \in D^{n}$. This means that for some $k(1 \leq k \leq n) c_{i}=d_{i}$ if $i \neq k$ and

$$
d_{k}= \begin{cases}a, & \text { if } c_{k}=0, \\ 1, & \text { if } c_{k}=b .\end{cases}
$$

Let $c_{i}=\left(u_{i}, v_{i}\right), d_{i}=\left(p_{i}, q_{i}\right)$. Then $u_{i} \leq p_{i}$ and $v_{i}=q_{i}$ for all $i=1, \ldots, n$. Therefore, $\left(u_{1}, \ldots, u_{n}, \bar{v}_{1}, \ldots, \bar{v}_{n}\right) \leq\left(p_{1}, \ldots, p_{n}, \bar{q}_{1}, \ldots, \bar{q}_{n}\right)$ and $\left(v_{1}, \ldots, v_{n}, \bar{u}_{1}, \ldots, \bar{u}_{n}\right) \geq\left(q_{1}, \ldots, q_{n}, \bar{p}_{1}, \ldots, \bar{p}_{n}\right)$. Hence,

$$
\varphi_{f}\left(u_{1}, \ldots, u_{n}, \bar{v}_{1}, \ldots, \bar{v}_{n}\right) \leq \varphi_{f}\left(p_{1}, \ldots, p_{n}, \bar{q}_{1}, \ldots, \bar{q}_{n}\right)
$$

and

$$
\varphi_{f}\left(v_{1}, \ldots, v_{n}, \bar{u}_{1}, \ldots, \bar{u}_{n}\right) \geq \varphi_{f}\left(q_{1}, \ldots, q_{n}, \bar{p}_{1}, \ldots, \bar{p}_{n}\right) .
$$

Thus, the first coordinate of $f(c)$ is less than (or equal to) the first coordinate of $f(d)$ and the second coordinate of $f(c)$ is greater than or equal to the second coordinate of $f(d)$. Thus, if $f(c)=0$ then $f(d) \in\{0, a\}$; if $f(c)=a$ then $f(d)=a$; and if $f(c)=1$ then $f(d) \in\{1, a\}$.

Corollary 2.5. There are $m_{2 n}$ De Morgan functions of $n$ variables.

\section{Free De Morgan algebras}

Denote the set of all De Morgan functions of $n$ variables by $\mathcal{D}_{n}$. For the functions $f, g: D^{n} \rightarrow D$ define $f+g, f \cdot g$ and $\bar{f}$ by the standard way, i.e. $(f+g)(x)=f(x)+g(x),(f \cdot g)(x)=f(x) \cdot g(x), \bar{f}(x)=\overline{f(x)}, x \in D^{n}$, where the operations on the right hand side are the operations of De Morgan algebra 4. We claim that $\mathcal{D}_{n}$ is closed under those operations, i.e. if $f, g \in \mathcal{D}_{n}$, then $f+g, f \cdot g, \bar{f} \in \mathcal{D}_{n}$. We can verify it straightforwardly, using the definition of De Morgan function. But it is easier to prove it, using Theorem 2.4. If $f, g \in \mathcal{D}_{n}$ then

$$
f\left(x_{1}, \ldots, x_{n}\right)=\left(\varphi_{f}\left(u_{1}, \ldots, u_{n}, \bar{v}_{1}, \ldots, \bar{v}_{n}\right), \varphi_{f}\left(v_{1}, \ldots, v_{n}, \bar{u}_{1}, \ldots, \bar{u}_{n}\right)\right),
$$

and

$$
g\left(x_{1}, \ldots, x_{n}\right)=\left(\varphi_{g}\left(u_{1}, \ldots, u_{n}, \bar{v}_{1}, \ldots, \bar{v}_{n}\right), \varphi_{g}\left(v_{1}, \ldots, v_{n}, \bar{u}_{1}, \ldots, \bar{u}_{n}\right)\right) .
$$

Hence:

$$
\begin{aligned}
& (f+g)\left(x_{1}, \ldots, x_{n}\right) \\
& \quad=\left(\left(\varphi_{f}+\varphi_{g}\right)\left(u_{1}, \ldots, u_{n}, \bar{v}_{1}, \ldots, \bar{v}_{n}\right),\left(\varphi_{f}+\varphi_{g}\right)\left(v_{1}, \ldots, v_{n}, \bar{u}_{1}, \ldots, \bar{u}_{n}\right)\right)
\end{aligned}
$$


and

$$
\begin{aligned}
& (f \cdot g)\left(x_{1}, \ldots, x_{n}\right) \\
& \quad=\left(\left(\varphi_{f} \cdot \varphi_{g}\right)\left(u_{1}, \ldots, u_{n}, \bar{v}_{1}, \ldots, \bar{v}_{n}\right),\left(\varphi_{f} \cdot \varphi_{g}\right)\left(v_{1}, \ldots, v_{n}, \bar{u}_{1}, \ldots, \bar{u}_{n}\right)\right) .
\end{aligned}
$$

As $\varphi_{f}, \varphi_{g}$ are monotone boolean functions, $\varphi_{f} \cdot \varphi_{g}$ and $\varphi_{f}+\varphi_{g}$ are monotone, as well. So by Theorem $2.4 f+g$ and $f \cdot g$ are De Morgan functions.

Further, from Lemma 1.5 we get:

$$
\begin{aligned}
\bar{f}\left(x_{1}, \ldots, x_{n}\right) & =\overline{\left(\varphi_{f}\left(u_{1}, \ldots, u_{n}, \bar{v}_{1}, \ldots, \bar{v}_{n}\right), \varphi_{f}\left(v_{1}, \ldots, v_{n}, \bar{u}_{1}, \ldots, \bar{u}_{n}\right)\right)} \\
& =\left(\overline{\varphi_{f}\left(v_{1}, \ldots, v_{n}, \bar{u}_{1}, \ldots, \bar{u}_{n}\right)}, \overline{\varphi_{f}\left(u_{1}, \ldots, u_{n}, \bar{v}_{1}, \ldots, \bar{v}_{n}\right)}\right) \\
& =\left(\varphi_{f}^{\prime}\left(u_{1}, \ldots, u_{n}, \bar{v}_{1}, \ldots, \bar{v}_{n}\right), \varphi_{f}^{\prime}\left(v_{1}, \ldots, v_{n}, \bar{u}_{1}, \ldots, \bar{u}_{n}\right)\right) .
\end{aligned}
$$

And $\varphi_{f}$ is monotone; therefore, $\varphi_{f}^{\prime}$ is also monotone. Hence, $\bar{f}$ is a De Morgan function.

Thus, we get an algebra: $\mathfrak{D}_{n}=\left(\mathcal{D}_{n},\left\{+, \cdot{ }^{-}, 0,1\right\}\right)$ (here 0 and 1 are the constant De Morgan functions), which obviously is a De Morgan algebra. Also, for $f, g \in \mathcal{D}_{n}$ we have: $\varphi_{f+g}=\varphi_{f}+\varphi_{g}, \varphi_{f \cdot g}=\varphi_{f} \cdot \varphi_{g}, \varphi_{\bar{f}}=\varphi_{f}^{\prime}$.

Let $a=\left(a_{1}, a_{2}\right), b=\left(b_{1}, b_{2}\right) \in 2^{\{1, \ldots, n\}} \times 2^{\{1, \ldots, n\}}$. We say that $a \subseteq$ $b$, if $a_{1} \subseteq b_{1}$ and $a_{2} \subseteq b_{2}$. In this way, we get a partially ordered set $2^{\{1, \ldots, n\}} \times 2^{\{1, \ldots, n\}}(\subseteq)$. For an antichain $S \subseteq 2^{\{1, \ldots, n\}} \times 2^{\{1, \ldots, n\}}$, define the function $f_{S}: D^{n} \rightarrow D$ in the following way:

$$
f_{S}\left(x_{1}, \ldots, x_{n}\right)=\sum_{s=\left(s_{1}, s_{2}\right) \in S}\left(\prod_{i \in s_{1}} x_{i} \cdot \prod_{i \in s_{2}} \bar{x}_{i}\right) .
$$

Notice that $f_{S}$ does not depend on the order of the elements in the set $S$ (cf. [32]).

Note that we set $f_{\emptyset}=0$ and $f_{\{(\emptyset, \emptyset)\}}=1$.

Let us consider the functions

$$
\delta_{n}^{i}\left(x_{1}, \ldots, x_{n}\right)=x_{i}, i=1, \ldots, n,
$$

as functions $D^{n} \rightarrow D$. Obviously, $\delta_{n}^{i}$ is a De Morgan function. And according to 3.1 , for any antichain $S \subseteq 2^{\{1, \ldots, n\}} \times 2^{\{1, \ldots, n\}}$, we have:

$$
f_{S}=\sum_{s=\left(s_{1}, s_{2}\right) \in S}\left(\prod_{i \in s_{1}} \delta_{n}^{i} \cdot \prod_{i \in s_{2}} \overline{\delta_{n}^{i}}\right) .
$$

Hence, $f_{S} \in \mathcal{D}_{n}$, i.e. $f_{S}$ is a De Morgan function for any antichain $S \subseteq$ $2^{\{1, \ldots, n\}} \times 2^{\{1, \ldots, n\}}$.

For $s=\left(s_{1}, s_{2}\right) \in 2^{\{1, \ldots, n\}} \times 2^{\{1, \ldots, n\}}$ let $s^{\prime}=s_{1} \cup\left\{n+i: i \in s_{2}\right\} \in 2^{\{1, \ldots, 2 n\}}$, and for $S \subseteq 2^{\{1, \ldots, n\}} \times 2^{\{1, \ldots, n\}}$ let $S^{\prime}=\left\{s^{\prime}: s \in S\right\} \subseteq 2^{\{1, \ldots, 2 n\}}$. In this way, we give a bijective mapping from the set of all antichains of $2^{\{1, \ldots, n\}} \times 2^{\{1, \ldots, n\}}(\subseteq)$ to the set of all antichains of $2^{\{1, \ldots, 2 n\}}(\subseteq)$. And so the number of all antichains of the partially ordered set $2^{\{1, \ldots, n\}} \times 2^{\{1, \ldots, n\}}(\subseteq)$ is $m_{2 n}$. 
Now, for any De Morgan function $f \in \mathcal{D}_{n}$ from Proposition 1.3 and Theorem 2.4, we conclude that there exists an antichain $S^{\prime} \subseteq 2^{\{1, \ldots, 2 n\}}$ such that:

$$
\begin{aligned}
f\left(x_{1}, \ldots, x_{n}\right)=\left(\varphi_{f}\left(u_{1}, \ldots, u_{n}, \bar{v}_{1}, \ldots, \bar{v}_{n}\right), \varphi_{f}\left(v_{1}, \ldots, v_{n}, \bar{u}_{1}, \ldots, \bar{u}_{n}\right)\right) \\
=\left(\sum_{s^{\prime} \in S^{\prime}}\left(\prod_{\substack{i \in s^{\prime} \\
1 \leq i \leq n}} u_{i} \cdot \prod_{\substack{i \in s^{\prime} \\
n+1 \leq i \leq 2 n}} \bar{v}_{i-n}\right), \sum_{s^{\prime} \in S^{\prime}}\left(\prod_{\substack{i \in s^{\prime} \\
1 \leq i \leq n}} v_{i} \cdot \prod_{\substack{i \in s^{\prime} \\
n+1 \leq i \leq 2 n}} \bar{u}_{i-n}\right)\right) \\
=\sum_{s^{\prime} \in S^{\prime}}\left(\prod_{\substack{i \leq s^{\prime} \\
1 \leq n}}\left(u_{i}, v_{i}\right) \cdot \prod_{\substack{i \in s^{\prime} \\
n+1 \leq i \leq 2 n}}\left(\bar{v}_{i-n}, \bar{u}_{i-n}\right)\right) \\
=\sum_{s=\left(s_{1}, s_{2}\right) \in S}\left(\prod_{i \in s_{1}} x_{i} \cdot \prod_{i \in s_{2}} \bar{x}_{i}\right)=f_{S}\left(x_{1}, \ldots, x_{n}\right),
\end{aligned}
$$

where $S$ is the antichain of $2^{\{1, \ldots, n\}} \times 2^{\{1, \ldots, n\}}(\subseteq)$, corresponding to $S^{\prime}$.

Moreover, the number of all De Morgan functions of $n$ variables is the same as the number of all antichains of $2^{\{1, \ldots, n\}} \times 2^{\{1, \ldots, n\}}(\subseteq)$. Hence, we get the following result.

THeOREM 3.1. For any De Morgan function $f$ of $n$ variables there exists a unique antichain $S \subseteq 2^{\{1, \ldots, n\}} \times 2^{\{1, \ldots, n\}}$ such that $f=f_{S}$.

In particular, $f_{S_{1}} \neq f_{S_{2}}$ if $S_{1} \neq S_{2}$.

Thus, every nonconstant De Morgan function can be uniquely presented in the form 3.1). This form is called the canonical form (or disjunctive normal form (or briefly - DNF)) of De Morgan function $f$. Notice that from Theorem 2.4 and from the proofs of Theorem 3.1 and Proposition 1.3. we get an algorithm which, given a De Morgan function, gives its disjunctive normal form. It is easy to see that the complexity of this algorithm is linear depending on the number of the rows of the table of the given De Morgan function $f$.

We can also prove that every nonconstant De Morgan function can be uniquely presented in conjunctive normal form $(\mathrm{CNF})$, i.e. in the following form:

$$
\prod_{\left(s_{1}, s_{2}\right) \in S}\left(\sum_{i \in s_{1}} x_{i}+\sum_{i \in s_{2}} \bar{x}_{i}\right) .
$$

THEOREM 3.2. The algebra $\mathfrak{D}_{n}$ is the free De Morgan algebra with the system of free generators: $\Delta=\left\{\delta_{n}^{1}, \ldots, \delta_{n}^{n}\right\}$. Hence, every free $n$-generated De Morgan algebra is isomorphic to the De Morgan algebra $\mathfrak{D}_{n}$.

Proof. Let $\mathfrak{F}=\left(Q ;\left\{+, \cdot,{ }^{-}, 0,1\right\}\right)$ be a De Morgan algebra and $\mu: \Delta \rightarrow Q$ be a mapping. We prove that there exists a unique homomorphism: $\nu: \mathcal{D}_{n} \rightarrow \mathfrak{F}$ 
with $\left.\nu\right|_{\Delta}=\mu$. Any element $f \in \mathcal{D}_{n}$ can be represented in the form

$$
f=\sum_{s=\left(s_{1}, s_{2}\right) \in S}\left(\prod_{i \in s_{1}} \delta_{n}^{i} \cdot \prod_{i \in s_{2}} \overline{\delta_{n}^{i}}\right),
$$

for the uniquely determined antichain $S \subseteq 2^{\{1, \ldots, n\}} \times 2^{\{1, \ldots, n\}}$. Set

$$
\nu(f)=\sum_{s=\left(s_{1}, s_{2}\right) \in S}\left(\prod_{i \in s_{1}} \mu\left(\delta_{n}^{i}\right) \cdot \prod_{i \in s_{2}} \overline{\mu\left(\delta_{n}^{i}\right)}\right) .
$$

Obviously, $\nu$ is a homomorphism and $\nu\left(\delta_{n}^{i}\right)=\mu\left(\delta_{n}^{i}\right), i=1, \ldots, n$. Uniqueness of $\nu$ is evident too.

A similar result is valid for the finitely generated free algebras of the hypervariety defined by the system of hyperidentities of De Morgan algebras (Boolean algebras, distributive lattices) $(25,30,32)$.

\section{Conclusion}

We give a new characterization of finitely generated free De Morgan algebras. The free De Morgan algebras have been characterized by several authors by describing the canonical forms of their elements $([2,4,13,15])$ (cf. 27, 28, 32]). Besides, it is commonly known that the free Boolean algebra on $n$ free generators is isomorphic to the Boolean algebra of Boolean functions of $n$ variables. In this paper, we introduce the concept of De Morgan function and prove that the free De Morgan algebra on $n$ free generators is isomorphic to the De Morgan algebra of De Morgan functions of $n$ variables. The advantage of this representation of the free De Morgan algebra is the fact that it gives rise to a new concept of the De Morgan function, which is a new object in discrete mathematics.

Acknowledgement. Thanks to the referee for useful remarks.

This research was supported by State Committee Science of Republic of Armenia.

\section{References}

[1] O. Arieli, A. Avron, The value of four values, Artificial Intelligence 102 (1998), 97-141.

[2] R. Balbes, P. Dwinger, Distributive lattices, Univ. of Missouri Press, 1974.

[3] N. D. Belnap, A useful for valued logic, in: G. Epstein, J. M. Dunn (Eds.), Modern Uses of Multiple-Valued Logic, Reidel Publishing Company, Boston, 1977, 7-73.

[4] J. Berman, W. Blok, Stipulations, multi-valued logic and De Morgan algebras, MultiValued Logic 7(5-6) (2001), 391-416.

[5] A. Białynicki-Birula, H. Rasiowa, On the representation of quasi-Boolean algebras, Bull. Acad. Polon. Sci. Ser. Math. Astronom. Phys. 5 (1957), 259-261.

[6] G. Birkhoff, Lattice Theory, 3rd Ed., American Mathematical Society, Providence, Rhode Island, 1967. 
[7] F. Bou, U. Rivieccio, The logic of distributive bilattices, Log. J. IGPL 19 (2011), $183-216$.

[8] J. A. Brzozowski, A characterization of De Morgan algebras, Internat. J. Algebra Comput. 11 (2001), 525-527.

[9] J. A. Brzozowski, De Morgan bisemilattices, Proceedings of the 30th IEEE International Symposium on Multiple-Valued Logic, (ISMVL 2000), May 23-25, (2000), p. 173.

[10] J. A. Brzozowski, Partially ordered structures for hazard detection, Special Session: The Many Lives of Lattice Theory, Joint Mathematics Meetings, San Diego, CA, January 6-9, (2002).

[11] Y. Crama, P. L. Hammer, Boolean Functions: Theory, Algorithms, and Applications, Cambridge University Press, New York, 2011.

[12] R. Dedekind, Über Zerlegungen von Zahlen durch ihre größten gemeinsamen Teiler, Festschrift der Techn. Hochsch. Braunschwig bei Gelegenheit der 69, Versammlung deutscher Naturforscher und Ärzte, (1897), 1-40.

[13] Z. Ésik, Free De Morgan Bisemigroups and Bisemilattices, Algebra Colloquium, Volume 10, Issue 1, June (2003), 23-32.

[14] M. Gehrke, C. Walker, E. Walker, A mathematical setting for fuzzy logics, Internat. J. Uncertain. Fuzziness Knowledge-Based Systems 5(3) (1997), 223-238.

[15] M. Gehrke, C. Walker, E. Walker, Some comments on fuzzy normal forms, Proceedings of the ninth IEEE International Conference on Fuzzy Systems, FUZZ-IEEE 2000, vol. 2, 593-598.

[16] G. Grätzer, Lattice Theory: Foundation, Springer, Basel AG, 2011.

[17] G. Grätzer, Universal Algebra, Springer-Verlag, 2010.

[18] J. A. Kalman, Lattices with involution, Trans. Amer. Math. Soc. 87 (1958), 485-491.

[19] L. H. Kauffman, De Morgan Algebras - completeness and recursion, Proceedings of the eighth International Symposium on Multiple-Valued Logic, IEEE Computer Society Press Los Alamitos, CA, USA, (1978), 82-86.

[20] M. Kondo, Characterization theorem of 4-valued De Morgan logic, Mem. Fac. Sci. Eng. Shimane Univ. Ser. B Math. Sci. 31 (1998), 73-80.

[21] A. D. Korshunov, Monotone Boolean functions, Uspekhi Mat. Nauk 58:5(353) (2003), 89-162. English translation in: Russian Math. Surveys 58(5) (2003), 929-1001.

[22] A. A. Markov, Constructive logic, Uspekhi Mat. Nauk 5 (1950), 187-188, (in Russian).

[23] B. Mobasher, D. Pigozzi, G. Slutzki, Multi-valued logic programming semantics, An algebraic approach, Theoret. Comput. Sci. 171 (1997), 77-109.

[24] G. C. Moisil, Recherches sur l'algebre de la logique, Annales scientifiques de l'Universite de Jassy 22 (1935), 1-117.

[25] Yu. M. Movsisyan, Introduction to the theory of algebras with hyperidentities, Yerevan State University Press, Yerevan, 1986, (in Russian).

[26] Yu. M. Movsisyan, Hyperidentities and hypervarieties in algebras, Yerevan State University Press, Yerevan, 1990, (in Russian).

[27] Yu. M. Movsisyan, Hyperidentities of Boolean algebras, Izv. Ross. Akad. Nauk Ser. Mat. 56 (1992), 654-672. English translation in: Russian Acad. Sci. Izv. Math. 40 (1993), 607-622.

[28] Yu. M. Movsisyan, Algebras with hyperidentities of the variety of Boolean algebras, Izv. Ross. Akad. Nauk Ser. Mat. 60 (1996), 127-168. English translation in: Russian Acad. Sci. Izv. Math. 60 (1996), 1219-1260. 
[29] Yu. M. Movsisyan, Hyperidentities in algebras and varieties, Uspekhi Mat. Nauk 53:1(319) (1998), 61-114. English translation in: Russian Math. Surveys 53(1) (1998), $57-108$.

[30] Yu. M. Movsisyan, Hyperidentities and hypervarieties, Sci. Math. Jpn. 54 (2001), 595-640.

[31] Yu. M. Movsisyan, Binary representations of algebras with at most two binary operations. A Cayley theorem for distributive lattices, Internat. J. Algebra Comput. 19(1) (2009), 97-106.

[32] Yu. M. Movsisyan, V. A. Aslanyan, Hyperidentities of De Morgan algebras, Log. J. IGPL 20 (2012), 1153-1174. doi:10.1093/jigpal/jzr053

[33] B. I. Plotkin, Universal Algebra, Algebraic Logic, and Databases, Kluwer Academic Publisher, 1994.

[34] H. P. Sankappanavar, A characterization of principal congruences of De Morgan algebras and its applications, Math. Logic in Latin America, Proc. IV Latin Amer. Symp. Math. Logic, Santiago, (1978), 341-349. North-Holland Pub. Co., Amsterdam, 1980.

[35] J. D. H. Smith, A. B. Romanowska, Post-Modern Algebra, A Wiley-Interscience Publication, John Wiley and Sons, Inc., New York, 1999.

[36] E. Sperner, Ein Satz über Untermengen einer endlichen Menge, Math. Z. 27 (1928), $544-548$.

Yu. M. Movsisyan, V. A. Aslanyan

DEPARTMENT OF MATHEMATICS AND MECHANICS

YEREVAN STATE UNIVERSITY

Alex Manoogian 1

YEREVAN 0025, ARMENIA

E-mail: yurimovsisyan@yahoo.com

vahagn.aslanyan@gmail.com

Received August 23, 2012; revised version April 8, 2013.

Communicated by A. Romanowska. 